\title{
OA-012 PREVALENCE AND RISK FACTORS OF VIROLOGICAL FAILURE AMONG CHILDREN ON ANTIRETROVIRAL THERAPY
}

Michael Owusu, ${ }^{1}$ Eric Mensah, ${ }^{2}$ Anthony Enimil, ${ }^{2}$ Mohamed Mutocheluhn ${ }^{2} .{ }^{1} K C C R$ Ghana; ${ }^{2}$ KNUST, Ghana

\subsection{6/bmjgh-2016-000260.20}

Background An unprecedented global effort at scaling up universal access to antiretroviral therapy has decreased the progression of HIV. However, due to challenges with supplies and adherence to intermittent antiretroviral therapy (ART) for mothers, infants continue to be infected, some with resistant viruses. Exposure to these resistant strains leads to nonresponsiveness to therapy resulting in virological failure. Children are more vulnerable to HIV drug resistance because of their life long treatment, the possible selection of resistant strains as a result of prophylaxis for mothers with HIV enrolled in PMTCT. The objective of the study was to determine the prevalence and risk factors of virological resistance among HIV-1-positive children on antiretroviral therapy.

Methods This was a longitudinal study that was performed at the HIV paediatric clinic of the Komfo Anokye Teaching Hospital, Kumasi, Ghana. Blood samples of children below the age of 18 years who had been on therapy for at least 3 months were analysed for virological load using real-time COBAS AmpliPrep/COBAS Taqman PCR. The samples were analysed at two consecutive time points when they came for their ART refill. Socio-demographic and clinical information was collected from their folders and also from the mother.

Results A total of 188 subjects were enrolled into the study from September 2015 to June 2016. The average duration on ART was 36 months (IQR=12-72 months). Of all subjects recruited, $134(71.3 \%)$ were found to be on regular drug ART. Of these, $21(15.7 \%)$ had virological failure and 102 (76.1\%) had virological suppression. A regression analysis showed that subjects whose parents were unemployed had 5.4 (1.4-20.9) chances of virological failure compared to those with parents employed.

Conclusions The risk of virological failure among HIV-positive children is still high. Efforts must be made to further identify the potential causes of virological failure among these children. 